\title{
Estrutura populacional de rebanho fechado da raça Nelore da linhagem Lemgruber
}

\author{
Priscila Silva Oliveira(1), Mário Luiz Santana Júnior ${ }^{(1)}$, Victor Breno Pedrosa(1), \\ Elisângela Chicaroni de Mattos Oliveira( ${ }^{(1)}$, Joanir Pereira Eler ${ }^{(1)}$ e José Bento Sterman Ferraz ${ }^{(1)}$
}

(1)Universidade de São Paulo, Faculdade de Zootecnia e Engenharia de Alimentos, Grupo de Melhoramento Animal e Biotecnologia, Caixa Postal 23, CEP 13635-970 Pirassununga, SP. E-mail: pri_med_vet@hotmail.com,10mario@gmail.com, vbpedrosa@yahoo.com.br, limattos@usp.br, joapeler@usp.br, jbferraz@usp.br

Resumo - O objetivo deste trabalho foi determinar a estrutura populacional e os efeitos causados pela endogamia, em um rebanho fechado da raça Nelore da linhagem Lemgruber. $\mathrm{O}$ arquivo de pedigree avaliado incluiu registros de 39.290 animais, 17.646 machos e 21.644 fêmeas, nascidos entre 1951 e 2007. A estrutura da população foi analisada com uso dos programas Poprep e Endog, tendo-se determinado algum nível de endogamia em $61,82 \%$ dos animais. O valor do F médio foi 3,02\% para toda a população e $4,89 \%$ para os animais endogâmicos; e o $\mathrm{F}$ máximo foi de $37,5 \%$. O número de ancestrais que contribuiu para a população referência foi 2.380 animais, dos quais apenas sete explicam $50 \%$ da variabilidade genética da população. $\mathrm{O}$ número efetivo de fundadores $\left(\mathrm{N}_{\mathrm{f}}\right)$ e o número efetivo de ancestrais $\left(\mathrm{N}_{\mathrm{a}}\right)$ nessa população foram 25 e 21, respectivamente. $\mathrm{O}$ incremento esperado de endogamia, causado pela contribuição desequilibrada dos fundadores, foi de $1,62 \%$. A estrutura populacional do rebanho apresenta envelhecimento dos reprodutores, com consequente aumento no intervalo de gerações, além de um contínuo incremento de endogamia e alta percentagem de indivíduos endogâmicos, fatos que comprometem o ganho genético anual e que devem merecer maior atenção dos selecionadores.

Termos para indexação: endogamia, Nelore Lemgruber, tamanho efetivo populacional, variabilidade genética.

\section{Population structure of a closed herd of Nellore cattle of the Lemgruber line}

\begin{abstract}
The objective of this work was to determine the population structure and the effects caused by inbreeding in a closed herd of Nellore cattle of the Lemgruber line. The pedigree file included records of 39,290 animal, 17,646 males and 21,644 females, born between 1951 and 2007. The population structure was analysed using the software Poprep and Endog, which resulted in some level of inbreeding for $61,82 \%$ of the animals. The average $\mathrm{F}$ value was $3.02 \%$ for the whole population and $4.89 \%$ for the inbred animals; and $\mathrm{F}$ maximum value was $37.5 \%$. The number of ancestors that contributed to the reference population was 2,380 animals, from which only seven explained $50 \%$ of the genetic variability of the population. The effective number of founders $\left(\mathrm{N}_{\mathrm{f}}\right)$ and the effective number of ancestors $\left(\mathrm{N}_{\mathrm{a}}\right)$ in this population were, respectively, 25 and 21. The expected increase of inbreeding, caused by the unbalanced contribution of founders, was $1.62 \%$. The herd populational structure shows the aging of the breeders, with a consequent increase of the generation's interval, as well as a continuous increase of inbreeding and a high percentage of inbred individuals. Such facts compromise the annual genetic gain and should deserve a closer attention from breeders.
\end{abstract}

Index terms: inbreeding, Nellore Lemgruber, effective population size, genetic variability.

\section{Introdução}

Os animais da raça Nelore da linhagem Lemgruber, muito conhecida por ser isolada dentro da raça, tem conquistado espaço na pecuária brasileira nos últimos anos, principalmente por meio da ampla utilização de alguns touros considerados geneticamente superiores, descendentes de uma pequena parcela de fundadores dessa linhagem. Atualmente, esses animais destacamse pela intensa contribuição ao rebanho nacional, além da alta rusticidade, precocidade no ganho de peso e na reprodução, habilidade materna e temperamento menos reativo.

O isolamento de uma população, principalmente daquelas submetidas a um constante programa de seleção, é considerado uma das principais causas do aumento da endogamia (Pedrosa et al., 2010). Nessas populações, teoricamente, não há introdução de animais oriundos de outros rebanhos, e aqueles considerados inapropriados para a reprodução são descartados. Assim, há redução da variabilidade genética, já que há uma redução do número de reprodutores com o 
descarte, o que propicia a fixação de alguns alelos. Esse fato acarreta um processo prejudicial para o progresso genético, pois impossibilita a identificação de animais superiores portadores de outros alelos.

O principal objetivo, ao se definir a estrutura dessas populações teoricamente isoladas, é estimar os parâmetros que as caracterizam, como a endogamia, o tamanho efetivo populacional e o intervalo de gerações, de modo que se possa direcionar o desenvolvimento de estratégias para um gerenciamento adequado dos recursos genéticos para a preservação e o aproveitamento da máxima diversidade.

Apesar de ser um processo prejudicial, tanto para a expressão das características de interesse econômico quanto para o ganho genético em programas de seleção, a endogamia é utilizada por alguns produtores com a finalidade de aumentar a prepotência nas linhagens (capacidade de um indivíduo de produzir descendentes semelhantes a ele), uma vez que indivíduos mais endogâmicos possuem menor variação de gametas e, assim, a progênie tende a ser mais uniforme. De acordo com Oliveira et al. (2002), é possível que grupos de animais, pertencentes a diferentes linhagens constituam, a cada dia, material genético distinto, com maior uniformidade dentro das linhagens. Assim, acasalamentos entre linhagens diferentes podem propiciar a manifestação da heterose, com o aumento do desempenho dos animais, decorrente da restauração da perda de vigor adaptativo ocorrida com a endogamia. Tal heterose, no entanto, será dependente da diferença entre as frequências gênicas das duas linhagens. Se as diferenças entre as frequências não forem significativas, o valor da heterose pode ser de pequena magnitude ou mesmo desprezível (Falconer \& Mackay, 1996).

O objetivo deste trabalho foi avaliar a estrutura populacional e os efeitos causados pela endogamia, em um rebanho fechado de bovinos da raça Nelore da linhagem Lemgruber.

\section{Material e Métodos}

Os arquivos de dados dos animais da raça Nelore, linhagem Lemgruber, foram obtidos junto ao Grupo de Melhoramento Animal e Biotecnologia (GMAB), da Faculdade de Zootecnia e Engenharia de Alimentos, da Universidade de São Paulo, em Pirassununga, SP. Os registros analisados no presente trabalho pertencem à Fazenda Mundo Novo, do Condomínio Agropecuário Irmãos Penteado Cardoso (Cipec Agropecuária), localizada no Km 125, da BR 050, em Uberaba (19²2'S $\left.48^{\circ} 13^{\prime} \mathrm{W}\right), \mathrm{MG}$.

$\mathrm{O}$ arquivo do pedigree utilizado nas análises incluiu registros de 39.290 animais, 17.646 machos e 21.644 fêmeas, nascidos entre 1951 e 2007. Além das informações genealógicas, disponíveis nos arquivos do GMAB, também foram utilizadas informações de ancestrais, fornecidas pela Associação Brasileira de Criadores de Zebuínos, o que permitiu obter estimativas mais precisas dos parâmetros que caracterizam a estrutura populacional. Nesse arquivo de pedigree, 34.691 animais possuíam ambos os pais conhecidos.

Os parâmetros populacionais foram calculados com utilização dos programas Poprep (Groeneveld et al., 2009) e Endog v.4.6 (Gutiérrez \& Goyache, 2005), que utilizam um conjunto mínimo de informações sobre cada animal para estimar os parâmetros relevantes que caracterizam a estrutura da população.

Esses programas estimam o coeficiente de endogamia individual (F) de acordo com Falconer \& Mackay (1996), em que F é igual ao relacionamento genético aditivo (f) entre seus pais, ou coeficiente de coancestralidade. Ou seja, $\mathrm{Fi}=\mathrm{f}_{\mathrm{sd}}$, em que i é o indivíduo, s é o pai e d é a mãe do indivíduo.

$\mathrm{O}$ tamanho efetivo de população $\mathrm{N}_{\mathrm{e}}$ também foi calculado de acordo com Falconer \& Mackay (1996), de duas maneiras distintas. Primeiro, utilizou-se a taxa de endogamia por geração, em que $N_{e}=1 / 2 \Delta F$ e $\Delta F=F_{t}-F_{t-1} / 1-F_{t-1}$, em que $F_{t}$ e $F_{t-1}$ representam a endogamia média da progênie e de seus pais, respectivamente. Em seguida, calculou-se o $\mathrm{N}_{\mathrm{e}}$ com base no número de reprodutores por geração, em que, $\mathrm{N}_{\mathrm{e}}=4 \mathrm{~N}_{\mathrm{m}} \mathrm{N}_{\mathrm{f}} / \mathrm{N}_{\mathrm{m}}+\mathrm{N}_{\mathrm{f}}$ e, $\mathrm{N}_{\mathrm{m}}$ e $\mathrm{N}_{\mathrm{f}}$ representam o número de reprodutores machos e fêmeas, respectivamente.

O intervalo de geração (IG) foi calculado segundo Falconer \& Mackay (1996), que o define como a média de idade dos pais ao nascimento de suas progênies mantidas para a reprodução. No cálculo do intervalo de geração, uma progênie foi considerada selecionada se deu origem a pelo menos um descendente. O resultado do IG foi computado por quatro caminhos distintos de seleção (pai-filho, pai-filha, mãe-filho e mãe-filha) por ano, para machos, fêmeas e para toda a população.

A integralidade do pedigree, que sintetiza a proporção de antepassados conhecidos em cada geração ascendente e quantifica a chance de detecção 
de endogamia no pedigree, foi calculada segundo Maccluer et al. (1983), pelas seguintes equações:

$$
\begin{gathered}
I_{d}=4 I_{d p a t} I_{d m a t} / 4 I_{d p a t}+I_{d m a t} \\
I_{d_{K}}=\frac{1}{d} \sum_{i=1}^{d} a_{i},
\end{gathered}
$$

em que: $\mathrm{K}$ representa a linha paterna (pat) ou materna (mat) de um indivíduo; $\mathrm{a}_{\mathrm{i}}$ é a proporção de ancestrais conhecidos na geração i; e d é o número de gerações consideradas no cálculo da integralidade do pedigree.

\section{Resultados e Discussão}

O número de ancestrais que contribuíram para a população de referência foi 2.380 animais, dos quais apenas sete explicaram $50 \%$ da variabilidade genética da população. $\mathrm{O}$ número efetivo de fundadores $\left(\mathrm{N}_{\mathrm{f}}\right)$, nessa população, foi de 25 , e o incremento esperado de endogamia causado pela contribuição desbalanceada dos fundadores foi de $1,62 \%$. O número efetivo de ancestrais $\left(\mathrm{N}_{\mathrm{a}}\right)$ observado foi igual a 21, muito inferior ao encontrado para a raça Indubrasil (107), por Vercesi Filho et al. (2002a). No entanto, valores semelhantes foram encontrados por Faria et al. (2010), para a raça Brahman (41 e 50,2), e por Vozzi et al. (2006), para a raça Nelore $(59,8)$ e Nelore Mocho $(61,5)$.

A razão entre o número efetivo de fundadores e ancestrais $\left(\mathrm{N}_{\mathrm{f}} / \mathrm{N}_{\mathrm{a}}\right)$ na população de referência foi de 1,19 , ou seja, nem todos os indivíduos apontados como fundadores dessa população foram efetivamente ancestrais dela; no entanto, esse resultado pode ser considerado satisfatório quando comparado a outros encontrados na literatura. De acordo com Faria et al. (2002), o valor médio para a razão $\mathrm{N}_{\mathrm{f}} / \mathrm{N}_{\mathrm{a}}$, encontrado para o Nelore Mocho, foi de 1,44. Reis Filho (2006) obteve para a raça Gir a razão de 1,95. Faria et al. (2010) relataram razão igual a 3,7, para o período de expansão da raça Brahman no Brasil (2002-2005), o que indica intenso uso de poucos reprodutores, provavelmente em consequência de predominância de inseminação artificial, produção in vitro e transferência de embriões.

A distribuição das matrizes quanto ao número de partos ao longo do tempo (Tabela 1) foi uma informação

\begin{tabular}{|c|c|c|c|c|c|c|c|c|c|c|c|c|c|c|c|c|}
\hline \multirow[t]{2}{*}{ Ano } & \multicolumn{16}{|c|}{ Distribuição das matrizes por número de partos } \\
\hline & 1 & 2 & 3 & 4 & 5 & 6 & 7 & 8 & 9 & 10 & 11 & 12 & 13 & 14 & 15 & $\geq 16$ \\
\hline 1983 & 662 & 239 & 95 & 33 & 7 & 3 & -- & & & & & & & & & \\
\hline 1984 & 697 & 570 & 209 & 78 & 28 & 3 & 2 & & & & & & & & & \\
\hline 1985 & 372 & 536 & 305 & 118 & 34 & 14 & 2 & -- & & & & & & & & \\
\hline 1986 & 269 & 374 & 367 & 166 & 70 & 15 & 7 & 1 & -- & & & & & & & \\
\hline 1987 & 417 & 317 & 473 & 369 & 152 & 69 & 15 & 6 & 1 & -- & & & & & & \\
\hline 1988 & 243 & 242 & 228 & 354 & 266 & 100 & 45 & 6 & 4 & 1 & -- & & & & & \\
\hline 1989 & 217 & 175 & 228 & 227 & 276 & 182 & 67 & 23 & 6 & 2 & 1 & & & & & \\
\hline 1990 & 270 & 169 & 181 & 191 & 206 & 196 & 103 & 47 & 11 & 4 & 1 & -- & & & & \\
\hline 1991 & 287 & 116 & 151 & 171 & 198 & 208 & 165 & 90 & 30 & 6 & 1 & 1 & -- & & & \\
\hline 1992 & 216 & 150 & 124 & 133 & 142 & 138 & 119 & 66 & 31 & 8 & -- & -- & 1 & -- & -- & \\
\hline 1993 & 239 & 189 & 126 & 125 & 118 & 128 & 105 & 64 & 34 & 11 & 1 & -- & -- & 1 & 1 & -- \\
\hline 1994 & 217 & 176 & 124 & 95 & 113 & 111 & 104 & 54 & 33 & 14 & 5 & 1 & -- & -- & -- & 1 \\
\hline 1995 & 261 & 171 & 151 & 150 & 107 & 108 & 98 & 102 & 52 & 27 & 11 & 5 & -- & -- & -- & 1 \\
\hline 1996 & 225 & 187 & 198 & 146 & 132 & 105 & 105 & 76 & 62 & 38 & 20 & 5 & 3 & -- & -- & -- \\
\hline 1997 & 208 & 157 & 160 & 131 & 111 & 115 & 84 & 79 & 65 & 33 & 22 & 16 & 4 & 2 & -- & -- \\
\hline 1998 & 273 & 267 & 147 & 194 & 157 & 121 & 104 & 81 & 65 & 49 & 27 & 17 & 6 & 2 & 1 & -- \\
\hline 1999 & 228 & 211 & 211 & 126 & 143 & 107 & 98 & 70 & 43 & 41 & 29 & 14 & 11 & 2 & 1 & -- \\
\hline 2000 & 223 & 142 & 182 & 155 & 126 & 122 & 87 & 83 & 60 & 45 & 27 & 11 & 10 & 9 & 1 & -- \\
\hline 2001 & 269 & 188 & 182 & 174 & 125 & 105 & 94 & 70 & 63 & 40 & 28 & 16 & 4 & 2 & 2 & -- \\
\hline 2002 & 221 & 173 & 87 & 82 & 75 & 53 & 56 & 45 & 29 & 26 & 16 & 8 & 5 & 2 & -- & -- \\
\hline 2003 & 149 & 180 & 196 & 131 & 116 & 90 & 69 & 72 & 55 & 38 & 25 & 15 & 9 & 6 & 1 & -- \\
\hline 2004 & 281 & 150 & 147 & 144 & 108 & 106 & 86 & 62 & 57 & 46 & 30 & 24 & 12 & 5 & 6 & 12 \\
\hline 2005 & 198 & 168 & 131 & 127 & 123 & 87 & 67 & 58 & 40 & 32 & 26 & 9 & 10 & 4 & 1 & -- \\
\hline 2006 & 247 & 220 & 131 & 120 & 113 & 109 & 84 & 59 & 49 & 35 & 25 & 16 & 12 & 7 & 4 & 4 \\
\hline 2007 & 205 & 123 & 171 & 100 & 114 & 91 & 88 & 64 & 47 & 36 & 23 & 16 & 3 & 7 & -- & -- \\
\hline
\end{tabular}

Tabela 1. Distribuição das matrizes da raça Nelore, linhagem Lemgruber, quanto à ordem de partos por ano. 
bastante importante, pois permitiu avaliar a taxa de reposição e de descarte das matrizes do rebanho. Verificou-se que, com o decorrer dos anos, não houve crescimento do rebanho por meio das novilhas, mas sim a manutenção de matrizes com um número de partos elevados, o que pode prejudicar tanto o ganho genético anual, quanto também elevar a taxa de endogamia, pois aumentam as chances de ocorrer acasalamentos entre indivíduos aparentados.

Demonstrou-se que poucos reprodutores deram origem a um grande número de filhos, em que os 50 primeiros ancestrais mais representativos, equivalentes a $6,7 \%$ dos touros, foram responsáveis por 58,73\% dos filhos nascidos neste rebanho (Tabela 2). Segundo Faria et al. (2002), quando um determinado número de animais é utilizado por mais tempo e deixa um número maior de filhos, além da influência sobre o intervalo de gerações, ocorre aumento na variância de tamanho de família, que é uma das principais causas da diminuição do tamanho efetivo da população.
A percentagem média de progênies dos touros e das matrizes selecionadas para se tornarem pais da geração seguinte foi de 72,86 e 16,94, respectivamente, e a proporção média touro:vaca foi de 1:13 (Tabela 3). No Brasil, a proporção touro:vaca adotada tradicionalmente pelos pecuaristas é de 1:25, o que resulta em subutilização dos touros, pois, segundo Santos et al. (2004), touros da raça Nelore em acasalamentos por monta natural podem suportar maior número de vacas,

Tabela 2. Descrição da contribuição individual e acumulada dos principais ancestrais da raça Nelore, linhagem Lemgruber.

\begin{tabular}{lcc}
\hline Ancestrais & $\begin{array}{c}\text { Contribuição } \\
\text { individual (\%) }\end{array}$ & $\begin{array}{c}\text { Contribuição } \\
\text { acumulada (\%) }\end{array}$ \\
\hline 1o (ZR7015) & 5,48 & 5,48 \\
2o (A1646) & 3,05 & 8,53 \\
3o (8107) & 3,03 & 11,56 \\
4o (ZRC0095) & 2,91 & 14,47 \\
5o (ZRB3194) & 2,46 & 16,93 \\
10 mais influentes & - & 25,44 \\
50 mais influentes & - & 58,73 \\
\hline
\end{tabular}

Tabela 3. Número de machos e fêmeas da raça Nelore, linhagem Lemgruber, na reprodução, por ano de nascimento da progênie.

\begin{tabular}{|c|c|c|c|c|c|}
\hline \multirow[t]{2}{*}{ Ano } & \multicolumn{2}{|c|}{ Machos } & \multicolumn{2}{|c|}{ Fêmeas } & \multirow[t]{2}{*}{ № de animais nascidos } \\
\hline & Machos com progênie & Com progênie selecionada ${ }^{(1)}$ & Fêmeas com progênie & Com progênie selecionada & \\
\hline 1983 & 25 & 19 & 1.034 & 202 & 1.054 \\
\hline 1984 & 24 & 20 & 1.583 & 153 & 1.595 \\
\hline 1985 & 26 & 21 & 1.361 & 207 & 1.429 \\
\hline 1986 & 37 & 26 & 1.254 & 152 & 1.278 \\
\hline 1987 & 50 & 33 & 1.748 & 187 & 1.836 \\
\hline 1988 & 50 & 42 & 1.488 & 186 & 1.504 \\
\hline 1989 & 44 & 29 & 1.384 & 233 & 1.415 \\
\hline 1990 & 44 & 39 & 1.371 & 215 & 1.391 \\
\hline 1991 & 63 & 44 & 1.378 & 235 & 1.434 \\
\hline 1992 & 56 & 40 & 1.114 & 211 & 1.136 \\
\hline 1993 & 50 & 34 & 1.121 & 225 & 1.147 \\
\hline 1994 & 53 & 33 & 1.026 & 209 & 1.053 \\
\hline 1995 & 65 & 55 & 1.156 & 248 & 1.256 \\
\hline 1996 & 61 & 52 & 1.216 & 229 & 1.331 \\
\hline 1997 & 62 & 47 & 1.089 & 211 & 1.193 \\
\hline 1998 & 69 & 60 & 1.393 & 296 & 1.514 \\
\hline 1999 & 69 & 51 & 1.269 & 251 & 1.341 \\
\hline 2000 & 75 & 47 & 1.217 & 217 & 1.288 \\
\hline 2001 & 79 & 52 & 1.309 & 229 & 1.367 \\
\hline 2002 & 57 & 46 & 854 & 202 & 883 \\
\hline 2003 & 62 & 48 & 1.084 & 219 & 1.156 \\
\hline 2004 & 75 & 58 & 1.185 & 225 & 1.298 \\
\hline 2005 & 84 & 15 & 1.055 & 23 & 1.083 \\
\hline 2006 & 82 & - & 1.214 & - & 1.237 \\
\hline 2007 & 90 & - & 1.074 & - & 1.091 \\
\hline
\end{tabular}

(1)Número de reprodutores que tiveram sua progênie selecionada em um determinado ano, para se tornarem pais da geração seguinte. 
o que certamente torna o sistema de acasalamento mais eficiente e econômico. De acordo com Fonseca (2000), a alteração nessa proporção tradicional para 1:60 e 1:80 acarreta redução no custo de cada bezerro desmamado de 18,6 e $22,8 \%$, respectivamente.

Os intervalos de gerações nas passagens pai-filho e pai-filha foram de 9,01 e 8,7 anos, respectivamente (Tabela 4), e podem ser considerados altos e similares aos demais encontrados para raças zebuínas criadas no Brasil (Faria et al., 2001, 2002; Vercesi Filho et al., 2002a, 2002b; Reis Filho, 2006). Essas elevadas estimativas são atribuídas, em parte, à rusticidade e adaptação da raça Nelore ao clima tropical, à elevada idade das vacas ao parto (sete anos em média) e à tardia substituição delas. A redução do ganho genético por unidade de tempo, com menor retorno econômico do programa, é a principal consequência dos longos intervalos de gerações.

O intervalo de geração médio de pai-progênie foi maior em relação às demais passagens gaméticas citadas (Tabela 4). Isso pode ser explicado pela elevada idade dos touros quando divulgado o resultado de sua primeira prova ou, ainda, pela contínua utilização de determinados reprodutores, sem rápida substituição por seus descendentes. Esses resultados diferem de outros descritos na literatura, em que as passagens de touro para progênie são menores do que as de matrizes para progênie (Toll \& Barker, 1979; Vassalo et al., 1986).

Quanto à análise do pedigree (Tabela 5), a estimativa deste parâmetro é de grande importância, uma vez que o coeficiente de endogamia de um indivíduo depende do conhecimento de sua ascendência. Assim, quanto mais se conhece a ascendência de um indivíduo, mais confiável será o seu coeficiente de endogamia estimado em relação à base populacional definida.

Para os animais nascidos nos últimos dez anos, os percentuais médios de pedigree completo, de acordo com o número de gerações conhecidas, foram: uma geração, 98,8\%; duas gerações, 98,4\%; três gerações, 97,4\%; quatro gerações, $88 \%$; cinco gerações, $88 \%$;

Tabela 4. Intervalo médio de geração(1) e número de observações por ano de nascimento, de animais da raça Nelore, linhagem Lemgruber.

\begin{tabular}{|c|c|c|c|c|c|c|c|c|c|c|}
\hline Ano & ss & Nss & $\mathrm{sd}$ & Nsd & ds & $\mathrm{Nds}$ & $\mathrm{dd}$ & Ndd & pop & Npop \\
\hline 1983 & 10,8 & 13 & 9,1 & 188 & 9,8 & 13 & 7,1 & 183 & 9,2 & 201 \\
\hline 1984 & 9,3 & 9 & 13,1 & 143 & 8,3 & 7 & 7,9 & 141 & 12,9 & 152 \\
\hline 1985 & 8,9 & 19 & 9,6 & 188 & 7,3 & 15 & 8,0 & 187 & 8,7 & 207 \\
\hline 1986 & 14,0 & 4 & 8,6 & 148 & 8,4 & 3 & 7,8 & 146 & 8,3 & 152 \\
\hline 1987 & 9,7 & 15 & 9,1 & 171 & 10,1 & 11 & 7,9 & 170 & 8,6 & 187 \\
\hline 1988 & 7,2 & 10 & 9,1 & 176 & 7,9 & 10 & 8,1 & 176 & 8,6 & 186 \\
\hline 1989 & 6,2 & 13 & 8,1 & 222 & 8,4 & 13 & 9,0 & 221 & 8,5 & 235 \\
\hline 1990 & 7,7 & 12 & 8,5 & 202 & 8,3 & 12 & 8,8 & 200 & 8,5 & 216 \\
\hline 1991 & 12,7 & 12 & 8,6 & 210 & 10,5 & 10 & 8,4 & 222 & 8,6 & 234 \\
\hline 1992 & 11,6 & 15 & 10,0 & 195 & 8,7 & 15 & 7,6 & 192 & 9,0 & 210 \\
\hline 1993 & 7,9 & 10 & 8,2 & 209 & 7,4 & 9 & 7,6 & 209 & 7,8 & 227 \\
\hline 1994 & 6,8 & 11 & 9,1 & 198 & 6,8 & 11 & 7,3 & 197 & 8,2 & 209 \\
\hline 1995 & 7,0 & 17 & 8,5 & 234 & 6,2 & 14 & 7,6 & 234 & 8,0 & 251 \\
\hline 1996 & 6,8 & 19 & 8,8 & 221 & 6,6 & 18 & 7,4 & 210 & 8,0 & 240 \\
\hline 1997 & 8,0 & 10 & 7,2 & 205 & 7,7 & 10 & 7,6 & 205 & 7,4 & 215 \\
\hline 1998 & 7,4 & 14 & 6,6 & 283 & 6,6 & 14 & 7,0 & 284 & 6,9 & 299 \\
\hline 1999 & 5,5 & 12 & 5,6 & 238 & 6,5 & 12 & 6,8 & 243 & 6,2 & 255 \\
\hline 2000 & 7,5 & 13 & 7,6 & 207 & 6,9 & 12 & 7,3 & 208 & 7,5 & 221 \\
\hline 2001 & 9,3 & 14 & 7,9 & 215 & 8,1 & 15 & 7,1 & 215 & 7,6 & 230 \\
\hline 2002 & 5,2 & 13 & 7,0 & 191 & 4,2 & 12 & 6,6 & 191 & 6,7 & 204 \\
\hline 2003 & 7,8 & 11 & 8,7 & 210 & 6,2 & 11 & 8,0 & 210 & 8,3 & 221 \\
\hline 2004 & 13,2 & 10 & 8,5 & 219 & 8,1 & 10 & 7,2 & 220 & 7,9 & 230 \\
\hline 2005 & 9,7 & 1 & 14,2 & 22 & 7,2 & 1 & 7,1 & 22 & 10,5 & 23 \\
\hline Média & 9,1 & - & 8,7 & - & 7,7 & - & 7,6 & - & 8,3 & - \\
\hline
\end{tabular}

${ }^{(1)}$ Média de idade dos pais, ao nascimento da progênie mantida para a reprodução. ss, pai para filho; Nss, número de machos para ss; sd, pai para filha; Nsd, número de fêmeas para sd; ds, mãe para filho; Nds, número de machos para ds; dd, mãe para filha; Ndd, número de fêmeas para dd; pop, intervalo médio de geração para toda a população; Npop, número de progênies selecionadas. 
seis gerações, 78,2\%. Esses dados são indicativos de que o controle de informações genealógicas do rebanho Nelore Lemgruber é de alta qualidade, com muitas gerações controladas.

Quanto à distribuição temporal da endogamia média na população (Figura 1), verificou-se que não foram observados valores acima de zero durante os primeiros anos. Provavelmente, isso aconteceu por se tratar de um período correspondente à formação do rebanho, em que os animais fundadores eram pouco aparentados

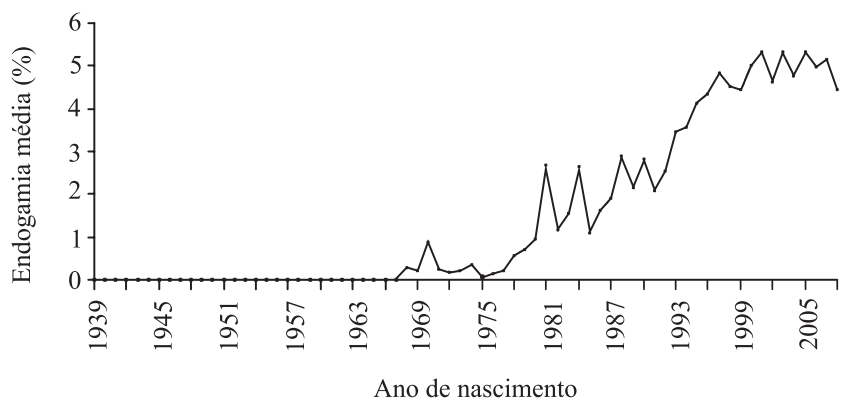

Figura 1. Endogamia média, por ano de nascimento, de animais da raça Nelore, linhagem Lemgruber. ou sua genealogia era desconhecida. Além disso, algumas informações de pedigree referentes aos animais desse período podem ter sido perdidas, o que impossibilitou o estabelecimento do parentesco entre eles. Nesse caso, sua recuperação e incorporação ao banco de dados traria grande contribuição ao estudo dessa população.

Com o decorrer dos anos, verificou-se que, assim como na percentagem de integralidade do pedigree, houve aumento no valor de F médio. Isso pode ser justificado pelo fato de que, quanto mais gerações completas de um indivíduo são conhecidas, maior a possibilidade de um ancestral importante aparecer diversas vezes no pedigree.

De acordo com Queiroz et al. (2000), o aumento de $\mathrm{F}$ ao longo das gerações, como o observado no presente estudo, pode ser explicado pela própria estrutura do rebanho, em que os animais fundadores são pouco aparentados ou de genealogia desconhecida. Com o passar do tempo, mediante a ocorrência de acasalamentos dentro do rebanho, aumenta o grau de parentesco entre os indivíduos, o que leva ao aumento da endogamia.

Tabela 5. Média da integralidade do pedigree (\%) por ano de nascimento, de animais da raça Nelore, linhagem Lemgruber, até a sexta geração.

\begin{tabular}{|c|c|c|c|c|c|c|c|}
\hline Ano & № de animais & $1^{\text {a }}$ ger. & 2⿳亠口冋 ger. & 3- ger. & 4a ger. & 5- ger. & $6^{a}$ ger. \\
\hline 1983 & 1.054 & 95,3 & 90,5 & 72,4 & 54,9 & 44,0 & 36,7 \\
\hline 1984 & 1.595 & 88,0 & 86,0 & 67,0 & 51,0 & 40,9 & 34,1 \\
\hline 1985 & 1.429 & 88,9 & 87,8 & 73,4 & 56,5 & 45,4 & 37,8 \\
\hline 1986 & 1.278 & 97,1 & 94,1 & 79,0 & 61,6 & 49,5 & 41,3 \\
\hline 1987 & 1.836 & 95,9 & 91,2 & 80,7 & 63,5 & 51,2 & 42,7 \\
\hline 1988 & 1.504 & 96,9 & 94,2 & 85,3 & 68,4 & 55,3 & 46,1 \\
\hline 1989 & 1.415 & 95,9 & 93,3 & 86,8 & 70,7 & 57,5 & 47,9 \\
\hline 1990 & 1.391 & 96,9 & 93,2 & 87,6 & 72,3 & 58,6 & 48,9 \\
\hline 1991 & 1.434 & 96,2 & 93,2 & 87,4 & 73,0 & 59,8 & 49,9 \\
\hline 1992 & 1.136 & 95,8 & 94,5 & 90,0 & 77,3 & 63,7 & 53,3 \\
\hline 1993 & 1.147 & 93,7 & 91,6 & 88,0 & 77,9 & 64,8 & 54,3 \\
\hline 1994 & 1.053 & 99,1 & 98,4 & 95,6 & 85,7 & 71,6 & 60,1 \\
\hline 1995 & 1.256 & 98,7 & 98,6 & 96,4 & 88,4 & 74,7 & 62,8 \\
\hline 1996 & 1.331 & 97,8 & 97,4 & 94,2 & 86,4 & 73,4 & 61,9 \\
\hline 1997 & 1.193 & 99,8 & 99,6 & 98,6 & 93,5 & 81,2 & 68,9 \\
\hline 1998 & 1.514 & 99,5 & 99,2 & 98,7 & 94,2 & 83,2 & 70,9 \\
\hline 1999 & 1.341 & 99,3 & 98,9 & 98,1 & 94,5 & 84,6 & 72,6 \\
\hline 2000 & 1.288 & 99,8 & 99,5 & 98,4 & 94,8 & 85,4 & 73,7 \\
\hline 2001 & 1.367 & 99,2 & 99,0 & 98,7 & 95,3 & 87,7 & 76,6 \\
\hline 2002 & 883 & 93,3 & 93,2 & 93,1 & 90,9 & 85,2 & 75,5 \\
\hline 2003 & 1.156 & 98,7 & 98,6 & 97,4 & 94,9 & 88,7 & 78,6 \\
\hline 2004 & 1.298 & 99,5 & 98,8 & 97,3 & 94,4 & 88,5 & 79,2 \\
\hline 2005 & 1.083 & 99,8 & 99,8 & 97,5 & 94,0 & 88,4 & 79,8 \\
\hline 2006 & 1.237 & 98,6 & 98,6 & 98,0 & 96,4 & 92,6 & 84,9 \\
\hline 2007 & 1.091 & 99,5 & 97,9 & 96,6 & 94,7 & 90,9 & 83,8 \\
\hline
\end{tabular}


Dos 39.290 animais que constam do pedigree, 24.291 $(61,82 \%)$ apresentaram algum nível de endogamia. $\mathrm{O}$ valor de $\mathrm{F}$ médio para toda a população e para os animais endogâmicos foi de 3,02, 4,89\%, respectivamente, e o $\mathrm{F}$ máximo encontrado foi de $37,5 \%$. Apesar de a endogamia média do rebanho não ser considerada alta, esses valores foram muito superiores aos observados por Santana Junior et al. (2010), que

Tabela 6. Número de indivíduos (N), coeficiente de endogamia médio (F), percentagem de indivíduos endogâmicos (End), coeficiente de endogamia médio dos endogâmicos $\left(\mathrm{F}_{\text {end }}\right)$ e tamanho efetivo da população $\left(\mathrm{N}_{\mathrm{e}}\right)$, por geração completa de animais da raça Nelore, linhagem Lemgruber.

\begin{tabular}{crrrrr}
\hline Geração & $\mathrm{N}$ & $\mathrm{F}(\%)$ & $\mathrm{End}(\%)$ & $\mathrm{F}_{\text {end }}(\%)$ & \multicolumn{1}{c}{$\mathrm{N}_{\mathrm{e}}$} \\
\hline 0 & 4.599 & 0,000 & 0,000 & 0,000 & - \\
1 & 3.509 & 0,135 & 3,078 & 4,375 & 371,3 \\
2 & 9.475 & 2,158 & 40,760 & 5,294 & 24,6 \\
3 & 12.456 & 3,860 & 89,539 & 4,311 & 28,7 \\
4 & 7.768 & 5,310 & 98,932 & 5,367 & 33,1 \\
5 & 1.457 & 5,749 & 100,000 & 5,749 & 107,8 \\
6 & 26 & 5,466 & 100,000 & 5,466 & - \\
\hline
\end{tabular}

trabalharam com reprodutores da raça Nelore criados no Brasil. Além disso, verificou-se aumento contínuo no coeficiente de endogamia médio e na percentagem de indivíduos endogâmicos com o transcorrer das gerações (Tabela 6). No entanto, esses resultados foram bastante semelhantes aos encontrados por Reis Filho (2006), ao realizar estudo sobre a estrutura populacional com animais da raça Gir.

$\mathrm{O}$ valor de $\mathrm{F}$ médio dos touros foi de 1,81 e de $1,57 \%$ para as matrizes, e a taxa de percentagem de acasalamento entre irmãos completos e entre pais e filhos, na população em questão, foi de 1,47 e $0,42 \%$, respectivamente (Tabela 7). O maior valor de $\mathrm{F}$ para os touros, em comparação ao $\mathrm{F}$ das matrizes, pode ser explicado pela maior ocorrência da pressão de seleção, em que a utilização de touros com valores genéticos mais elevados, para as características economicamente importantes, tende a aumentar os laços genéticos entre os reprodutores.

A oscilação observada no $\mathrm{N}_{\mathrm{e}}$ (Tabela 8) resulta do desequilíbrio existente entre o número de touros e matrizes, pois, quanto maior a diferença entre eles

Tabela 7. Número total de indivíduos e de indivíduos endogâmicos (End), e endogamia média dos indivíduos endogâmicos (F médio) da raça Nelore, linhagem Lemgruber.

\begin{tabular}{|c|c|c|c|c|c|c|c|c|c|}
\hline \multirow[t]{2}{*}{ Ano } & \multicolumn{3}{|c|}{ Animais } & \multicolumn{3}{|c|}{ Touros } & \multicolumn{3}{|c|}{ Matrizes } \\
\hline & Total & End & F médio & Total & End & F médio & Total & End & F médio \\
\hline 1983 & 1.054 & 305 & 0,0154 & 25 & 8 & 0,0443 & 1.034 & 46 & 0,0029 \\
\hline 1984 & 1.595 & 482 & 0,0263 & 24 & 7 & 0,0280 & 1.583 & 83 & 0,0031 \\
\hline 1985 & 1.429 & 400 & 0,0111 & 26 & 5 & 0,0150 & 1.361 & 107 & 0,0047 \\
\hline 1986 & 1.278 & 447 & 0,0164 & 37 & 11 & 0,0228 & 1.254 & 118 & 0,0052 \\
\hline 1987 & 1.836 & 773 & 0,0189 & 50 & 14 & 0,0139 & 1.748 & 179 & 0,0051 \\
\hline 1988 & 1.504 & 915 & 0,0290 & 50 & 17 & 0,0140 & 1.488 & 203 & 0,0071 \\
\hline 1989 & 1.415 & 878 & 0,0216 & 44 & 18 & 0,0161 & 1.384 & 208 & 0,0071 \\
\hline 1990 & 1.391 & 873 & 0,0283 & 44 & 18 & 0,0140 & 1.371 & 273 & 0,0097 \\
\hline 1991 & 1.434 & 877 & 0,0208 & 63 & 25 & 0,0141 & 1.378 & 341 & 0,0108 \\
\hline 1992 & 1.136 & 781 & 0,0255 & 56 & 24 & 0,0161 & 1.114 & 394 & 0,0148 \\
\hline 1993 & 1.147 & 881 & 0,0346 & 50 & 20 & 0,0133 & 1.121 & 439 & 0,0168 \\
\hline 1994 & 1.053 & 898 & 0,0357 & 53 & 27 & 0,0151 & 1.026 & 463 & 0,0158 \\
\hline 1995 & 1.256 & 1.125 & 0,0414 & 65 & 36 & 0,0187 & 1.156 & 580 & 0,0187 \\
\hline 1996 & 1.331 & 1.203 & 0,0435 & 61 & 39 & 0,0246 & 1.216 & 675 & 0,0203 \\
\hline 1997 & 1.193 & 1.144 & 0,0484 & 62 & 40 & 0,0264 & 1.089 & 640 & 0,0209 \\
\hline 1998 & 1.514 & 1.462 & 0,0453 & 69 & 45 & 0,0263 & 1.393 & 928 & 0,0241 \\
\hline 1999 & 1.341 & 1.312 & 0,0443 & 69 & 51 & 0,0282 & 1.269 & 907 & 0,0270 \\
\hline 2000 & 1.288 & 1.258 & 0,0502 & 75 & 57 & 0,0303 & 1.217 & 891 & 0,0276 \\
\hline 2001 & 1.367 & 1.341 & 0,0534 & 79 & 63 & 0,0343 & 1.309 & 1.046 & 0,0314 \\
\hline 2002 & 883 & 820 & 0,0464 & 57 & 46 & 0,0349 & 854 & 736 & 0,0350 \\
\hline 2003 & 1.156 & 1.134 & 0,0532 & 62 & 51 & 0,0357 & 1.084 & 952 & 0,0359 \\
\hline 2004 & 1.298 & 1.243 & 0,0478 & 75 & 60 & 0,0355 & 1.185 & 1.059 & 0,0381 \\
\hline 2005 & 1.083 & 1.071 & 0,0533 & 84 & 66 & 0,0343 & 1.055 & 967 & 0,0382 \\
\hline 2006 & 1.237 & 1.216 & 0,0499 & 82 & 70 & 0,0354 & 1.214 & 1.135 & 0,0415 \\
\hline 2007 & 1.091 & 1.029 & 0,0516 & 90 & 80 & 0,0386 & 1.074 & 1.015 & 0,0404 \\
\hline
\end{tabular}


Tabela 8. Tamanho efetivo populacional $\left(\mathrm{N}_{\mathrm{e}}\right)$ de animais da raça Nelore, linhagem Lemgruber, calculado com base no número de touros e matrizes, e na taxa de endogamia $(\Delta \mathrm{F})$, por ano de nascimento.

\begin{tabular}{|c|c|c|c|c|c|c|}
\hline \multirow[t]{2}{*}{ Ano } & \multicolumn{4}{|c|}{ F médio } & \multirow[t]{2}{*}{$\Delta \mathrm{F}$} & \multirow[t]{2}{*}{$\mathrm{N}_{\mathrm{e}}$} \\
\hline & Progênie & Touro & Matriz & Pais & & \\
\hline & \multicolumn{6}{|c|}{$\mathrm{N}_{\mathrm{e}}$ calculado com base no número de touros e matrizes } \\
\hline 1983 & 1.054 & 25 & 1.034 & 1.059 & - & 98 \\
\hline 1984 & 1.595 & 24 & 1.583 & 1.607 & - & 95 \\
\hline 1985 & 1.429 & 26 & 1.361 & 1.387 & - & 102 \\
\hline 1986 & 1.278 & 37 & 1.254 & 1.291 & - & 144 \\
\hline 1987 & 1.836 & 50 & 1.748 & 1.798 & - & 194 \\
\hline 1988 & 1.504 & 50 & 1.488 & 1.538 & - & 193 \\
\hline 1989 & 1.415 & 44 & 1.384 & 1.428 & - & 171 \\
\hline 1990 & 1.391 & 44 & 1.371 & 1.415 & - & 171 \\
\hline 1991 & 1.434 & 63 & 1.378 & 1.441 & - & 241 \\
\hline 1992 & 1.136 & 56 & 1.114 & 1.170 & - & 213 \\
\hline 1993 & 1.147 & 50 & 1.121 & 1.171 & - & 191 \\
\hline 1994 & 1.053 & 53 & 1.026 & 1.079 & - & 202 \\
\hline 1995 & 1.256 & 65 & 1.156 & 1.221 & - & 246 \\
\hline 1996 & 1.331 & 61 & 1.216 & 1.277 & - & 232 \\
\hline 1997 & 1.193 & 62 & 1.089 & 1.151 & - & 235 \\
\hline 1998 & 1.514 & 69 & 1.393 & 1.462 & - & 263 \\
\hline 1999 & 1.341 & 69 & 1.269 & 1.338 & - & 262 \\
\hline 2000 & 1.288 & 75 & 1.217 & 1.292 & - & 283 \\
\hline 2001 & 1.367 & 79 & 1.309 & 1.388 & - & 298 \\
\hline 2002 & 883 & 57 & 854 & 911 & - & 214 \\
\hline 2003 & 1.156 & 62 & 1.084 & 1.146 & - & 235 \\
\hline 2004 & 1.298 & 75 & 1.185 & 1.260 & - & 282 \\
\hline 2005 & 1.083 & 84 & 1.055 & 1.139 & - & 311 \\
\hline 2006 & 1.237 & 82 & 1.214 & 1.296 & - & 307 \\
\hline \multirow[t]{2}{*}{2007} & 1.091 & 90 & 1.074 & 1.164 & - & 332 \\
\hline & \multicolumn{6}{|c|}{$\mathrm{N}_{\mathrm{e}}$ calculado com base na taxa de endogamia $(\Delta \mathrm{F})$} \\
\hline 1983 & 0,0154 & 0,0443 & 0,0029 & 0,0038 & 0,0116 & 43 \\
\hline 1984 & 0,0263 & 0,0280 & 0,0031 & 0,0035 & 0,0229 & 22 \\
\hline 1985 & 0,0111 & 0,0150 & 0,0047 & 0,0049 & 0,0062 & 81 \\
\hline 1986 & 0,0164 & 0,0228 & 0,0052 & 0,0057 & 0,0108 & 46 \\
\hline 1987 & 0,0189 & 0,0139 & 0,0051 & 0,0054 & 0,0136 & 37 \\
\hline 1988 & 0,0290 & 0,0140 & 0,0071 & 0,0073 & 0,0219 & 23 \\
\hline 1989 & 0,0216 & 0,0161 & 0,0071 & 0,0074 & 0,0143 & 35 \\
\hline 1990 & 0,0283 & 0,0140 & 0,0097 & 0,0098 & 0,0187 & 27 \\
\hline 1991 & 0,0208 & 0,0141 & 0,0108 & 0,0109 & 0,0100 & 50 \\
\hline 1992 & 0,0255 & 0,0161 & 0,0148 & 0,0148 & 0,0109 & 46 \\
\hline 1993 & 0,0346 & 0,0133 & 0,0168 & 0,0166 & 0,0183 & 27 \\
\hline 1994 & 0,0357 & 0,0151 & 0,0158 & 0,0157 & 0,0203 & 25 \\
\hline 1995 & 0,0414 & 0,0187 & 0,0187 & 0,0187 & 0,0231 & 22 \\
\hline 1996 & 0,0435 & 0,0246 & 0,0203 & 0,0205 & 0,0235 & 21 \\
\hline 1997 & 0,0484 & 0,0264 & 0,0209 & 0,0212 & 0,0278 & 18 \\
\hline 1998 & 0,0453 & 0,0263 & 0,0241 & 0,0242 & 0,0216 & 23 \\
\hline 1999 & 0,0443 & 0,0282 & 0,0270 & 0,0271 & 0,0177 & 28 \\
\hline 2000 & 0,0502 & 0,0303 & 0,0276 & 0,0278 & 0,0230 & 22 \\
\hline 2001 & 0,0534 & 0,0343 & 0,0314 & 0,0316 & 0,0225 & 22 \\
\hline 2002 & 0,0464 & 0,0349 & 0,0350 & 0,0350 & 0,0118 & 42 \\
\hline 2003 & 0,0532 & 0,0357 & 0,0359 & 0,0359 & 0,0179 & 28 \\
\hline 2004 & 0,0478 & 0,0355 & 0,0381 & 0,0380 & 0,0102 & 49 \\
\hline 2005 & 0,0533 & 0,0343 & 0,0382 & 0,0379 & 0,0160 & 31 \\
\hline 2006 & 0,0499 & 0,0354 & 0,0415 & 0,0411 & 0,0092 & 54 \\
\hline 2007 & 0,0516 & 0,0386 & 0,0404 & 0,0403 & 0,0118 & 42 \\
\hline
\end{tabular}

em um determinado ano, menor será o $\mathrm{N}_{\mathrm{e}}$. Logo, essa oscilação é resultante da variação na taxa de incremento da endogamia ao longo dos anos. Quanto maior o incremento de endogamia, menor é o $\mathrm{N}_{\mathrm{e}}$.

Essas observações apontam para a necessidade de intervenção na seleção dos indivíduos utilizados para reprodução, de modo que se possa evitar problemas futuros, como a redução no limite de seleção em decorrência das perdas na variabilidade genética, causadas pelo aumento da endogamia.

\section{Conclusões}

1. A estrutura populacional do rebanho de bovinos da raça Nelore, da linhagem Lemgruber estudada, é indicativa de envelhecimento da população de reprodutores e matrizes, com consequente intervalo de gerações elevado, o que compromete o ganho genético por ano.

2. O rebanho analisado apresenta redução da variabilidade genética, em razão do aumento contínuo do coeficiente de endogamia e do número de animais endogâmicos e aponta para a necessidade de direcionar os acasalamentos que darão origem às próximas gerações.

\section{Agradecimentos}

À Coordenação de Aperfeiçoamento de Pessoal de Nível Superior e ao Grupo de Melhoramento Genético Animal e Biotecnologia, da Faculdade de Zootecnia e Engenharia de Alimentos da Universidade de São Paulo, pelo apoio financeiro.

\section{Referências}

FALCONER, D.S.; MACKAY, T.F.C. Introduction to quantitative genetics. Harlow: Longman Group, 1996. 464p.

FARIA, F.J.C.; VERCESI FILHO, A.E.; MADALENA, F.E.; JOSAHKIAN, L.A. Parâmetros populacionais do rebanho Gir Mocho registrado no Brasil. Revista Brasileira de Zootecnia, v.30, p.1984-1988, 2001.

FARIA, F.J.C.; VERCESI FILHO, A.E.; MADALENA, F.E.; JOSAHKIAN, L.A. Estrutura populacional da raça Nelore Mocho. Arquivo Brasileiro de Medicina Veterinária e Zootecnia, v.54, p.501-509, 2002.

FARIA, L.C. de; QUEIROZ, S.A. de; VOZZI, P.A.; LÔBO, R.B.; MAGNABOSCO, C. de U.; OLIVEIRA, J.A. de. Variabilidade genética da raça Brahman no Brasil detectada por meio de análise de 
pedigree. Pesquisa Agropecuária Brasileira, v.45, p.1133-1140, 2010.

FONSECA, V.O.; FRANCO, C.S.; BERGMANN, J.A.G. Potencial reprodutivo e econômico de touros Nelore acasalados coletivamente na proporção de um touro para 80 vacas. Arquivo Brasileiro de Medicina Veterinária e Zootecnia, v.52, p.77-82, 2000.

GROENEVELD, E.; WESTHUIZEN, B.D.; MAIWASHE, A.; VOORDEWIND, F.; FERRAZ, J.B. POPREP: a generic report for population management. Genetics and Molecular Research, v.8, p.1158-1178, 2009.

GUTIÉRREZ, J.P.; GOYACHE, F. A note on ENDOG: a computer program for analyzing pedigree information. Journal of Animal Breeding and Genetics, v.122, p.172-176, 2005.

MACCLUER, J.W.; BOYCE, A.J.; DYKE, B.; WEITKAMP, L.R.; PFENNING, D.W.; PARSONS, C.J. Inbreeding and pedigree structure in Standardbred horses. Journal of Heredity, v.74, p.394-399, 1983.

OLIVEIRA, J.H.F. de; MAGNABOSCO, C. de U.; BORGES, A.M. de S.M. Nelore: base genética e evolução seletiva no Brasil. Planaltina: -se Cerrados, 2002. 54p. (-se Cerrados. Documentos, 49).

PEDROSA, V.B.; SANTANA JUNIOR, M.L.; OLIVEIRA, P.S.; ELER, J.P.; FERRAZ, J.B.S. Population structure and inbreeding effects on growth traits of Santa Inês sheep in Brazil. Small Ruminant Research, v.93, p.135-139, 2010.

QUEIROZ, S.A. de; ALBUQUERQUE, L.G. de; LANZONI, N.A. Efeito da endogamia sobre características de crescimento de bovinos da raça Gir no Brasil. Revista Brasileira de Zootecnia, v.29, p.1014-1019, 2000.
REIS FILHO, J.C. Endogamia na raça Gir. 2006. 61p. Dissertação (Mestrado) - Universidade Federal de Viçosa, Viçosa.

SANTANA JUNIOR, M.L.; OLIVEIRA, P.S.; PEDROSA, V.B.; ELER, J.P.; GROENEVELD, E.; FERRAZ, J.B.S. Effect of inbreeding on growth and reproductive traits of Nellore cattle in Brazil. Livestock Science, v.131, p.212-217, 2010.

SANTOS, M.D.; TORRES, C.A.A.; RUAS, J.R.M.; GUIMARÃES, J.D.; SILVA FILHO, J.M. Potencial reprodutivo de touros da raça Nelore submetidos a diferentes proporções touro: vaca. Arquivo Brasileiro de Medicina Veterinária e Zootecnia, v.56, p.497-503, 2004.

TOLL, G.L.; BARKER, J.S.F. Genetic history of the pedigree Poll Hereford breed in Australia: effect of the importation ban. Australian Journal of Agricultural Research, v.30, p.767-777, 1979.

VASSALO, J.M.; DIAZ, C.; GARCIA-MEDINA, J. R. A note on the population structure of the Avileña breed of cattle in Spain. Livestock Production Science, v.15, p.285-288, 1986.

VERCESI FILHO, A.E.; FARIA, F.J.C.; MADALENA, F.E.; JOSAHKIAN, L.A. Estrutura populacional do rebanho Indubrasil registrado no Brasil. Archivo Latinoamericano de Producción Animal, v.10, p.86-92, 2002a.

VERCESI FILHO, A.E.; FARIA, F.J.C.; MADALENA, F.E.; JOSAHKIAN, L.A. Estrutura populacional do rebanho Tabapuã registrado no Brasil. Arquivo Brasileiro de Medicina Veterinária e Zootecnia, v.54, p.609-617, 2002b.

VOZZI, P.A.; MARCONDES, C.R.; MAGNABOSCO, C. de U.; BEZERRA, L.A.F.; LÔBO, R.B. Structure and genetic variability in Nellore (Bos indicus) cattle by pedigree analysis. Genetics and Molecular Biology, v.29, p.482-485, 2006.

Recebido em 8 de dezembro de 2010 e aprovado em 2 de maio de 2011 